\title{
Design of CRLH Millimeter-Wave Passive Filters in Standard CMOS Process
}

\author{
Y. Zhang, N. H. W. Fong and N. Wong \\ Department of Electrical and Electronic Engineering, The University of Hong Kong \\ Pokfulam Road, Hong Kong \\ Email: \{yzhang,nfong,nwong\}@eee.hku.hk
}

\begin{abstract}
A novel approach to design millimeter-wave passive filters is presented using composite right/left-handed (CRLH) structures in a standard CMOS process. The filter is composed of left-handed open split-ring resonators (OSRR) or open complementary split-ring resonators (OCSRR). Such approach leads to a significant reduction in the on-chip area of the filter. It is also straightforward to construct similar filters based on the resonators' equivalent circuit models.
\end{abstract}

\section{INTRODUCTION}

With the development of ultra-high-speed and high-capacity point-to-point communication systems in recent years, the millimeter band has attracted increasing interests from communication system providers and researchers. Millimeter band offers the benefits of the higher guaranteed data rates of any wireless technology, longer distance transmissions and more cost effectiveness than optic systems.

Passive filter is one of the building blocks in low-power and high-selectivity millimeter band transceiver systems. For example, after pick-up by antenna, the signal has to go through a bandpass filter (BPF) to remove the out-of-band noise in order to increase the system sensitivity. This filter is usually built by discrete passive components subject to high selectivity requirement. If it can be integrated on a CMOS silicon chip, the cost of the whole transceiver can be significantly reduced.

In order to achieve the integration, geometry miniaturization is required. Some of millimeter CMOS passive filters have been developed with different folded structures [1-3]. However, in [1] and [2], the selectivity of the filters is inadequate and the bandwidth is too wide. [3] has a better selectivity but the size is large $\left(1.148 \times 1.49 \mathrm{~mm}^{2}\right)$. In addition, since all of them employ anomalous folded structures, their equivalent circuits are difficult to extract. This makes them hard to be transplanted to new filters on other frequency bands.

Recently, composite right/left-handed (CRLH) structures have drawn more and more attention. Since at resonance, their electrical sizes are much smaller than the signal wavelength, they offer a new approach where miniaturization and compatibility with CMOS technology are satisfied simultaneously [4].

The main purpose of this paper is to develop millimeter-wave filters by CRLH structures, with integration on a standard $0.13 \mu \mathrm{m}$ CMOS process. In Section II, the CRLH concept is reviewed and the basic resonator structures, namely, open split-ring resonator (OSRR) and open complementary split-ring resonator (OCSRR), are presented. By means of a lumped element circuit model, the structures could be simplified and easily analyzed. In section III, an example CRLH BPF layout and the corresponding simulation results are given. The performances are also compared with other reported structures. It is clear that CMOS millimeter-wave filters could be achieved based on CRLH structures. The small dimensions of the unit cells make this approach feasible for the integration with other circuits in a single chip.

\section{ANALYSIS OF CRLH RESONATORS}

\section{A. Concept of $C R L H$}

In the late $1960 \mathrm{~s}$, the concept of metamaterial, or referred to as left-handed $(\mathrm{LH})$ material, was first introduced. It is an artificial material characterized by negative values of permittivity $(\varepsilon)$ and permeability $(\mu)$ at specific frequencies. Since then, a lot of structures have been created based on this concept within the electronics and physics research communities. Actually, on the 2-D plane, the electromagnetic properties of $\mathrm{LH}$ materials can be explained with a general transmission line (TL) theory. As shown in Fig. 1(a), the left-handed TL model is the combination of a times-unit length series capacitance $C_{L}^{\prime}$ and a times-unit length shunt inductance $L_{L}^{\prime}$. On the other hand, the model of right-handed TL, shown in Fig. 1(b), can be represented as the combination of a per-unit length series inductance $L_{R}^{\prime}$ and a per-unit length shunt capacitance $C_{R}^{\prime}$ which is the dual of the left-handed TL. In general, the commonly mentioned CRLH structure represents the most general form of a structure with $\mathrm{LH}$ and $\mathrm{RH}$ attributes. The reader is referred to [5] for the theory of CRLH. 


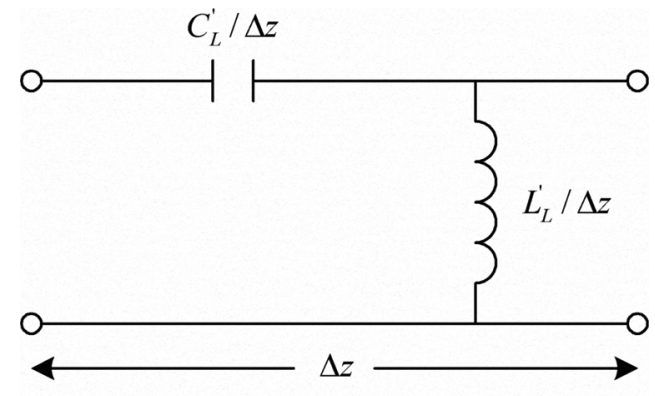

(a)

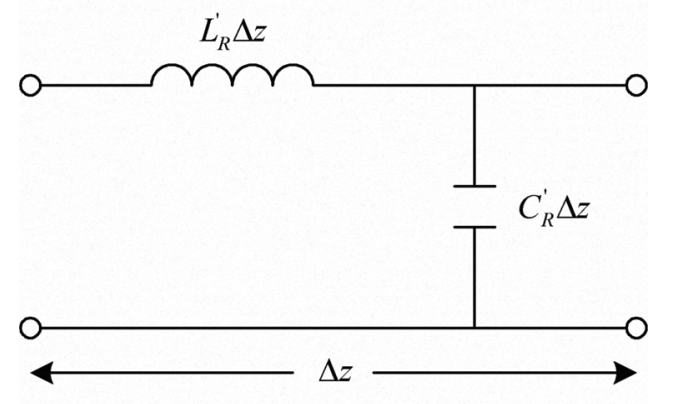

(b)

Fig. 1. Equivalent circuit models: (a) LH TL (b) RH TL.

As shown in Fig. 1, a purely RH TL and a purely LH TL exhibit lowpass and highpass properties, respectively. Therefore, by proper combination of RH and LH TLs, a resonator can be implemented by CRLH structure, and analyzed by transmission line theory. In fact, most of the CRLH structures built by now rely explicitly on the split-ring resonance to express a negative magnetic permeability to achieve a negative index of refraction.

\section{B. Conventional OSRR and OCSRR resonators}

In this work, the resonators of the filters are implemented by CRLH TLs combining OSRRs or OCSRRs. The conventional OSRR is shown in Fig. 2(a). Two metallic rings are printed on the dielectric substrate in parallel. One of the rings is excited by the current flowing through the signal conductor of the line, while the other ring is excited by the displacement current flowing through the slot between both rings. The structure of OCSRR is shown in Fig. 2(b). The OCSRR is the complementary counterpart of the OSRR by etching the same structure on the metal plane. The structure is able to inhibit signal propagation in the vicinity of their resonance frequency.

As was discussed in [6], the OSRR can be modeled as a series $\mathrm{LC}$ resonator, where $\mathrm{L}_{\mathrm{S}}$ is the inductance of a closed ring of averaged radius $r_{o}=r_{e x t}-c-d / 2$ and $\mathrm{C}_{0}$ is the distributed capacitance between the inner and outer rings. The OCSRR is the complementary counterpart of the OSRR, and it can be modeled as an open parallel resonant tank, where $\mathrm{L}_{0}$ is the inductance of the metallic strip between the ring slots, and $\mathrm{C}_{\mathrm{c}}$ is the capacitance of a disk of radius $r_{o}-c / 2$ surrounded by a metallic plane at a distance $c$ of its edge [6]. Since OCSRRs and OSRRs are complementary structures, it is expected that their resonance frequencies are roughly the same if they have identical dimensions and substrates, which have been theoretically demonstrated and experimentally validated in [6].
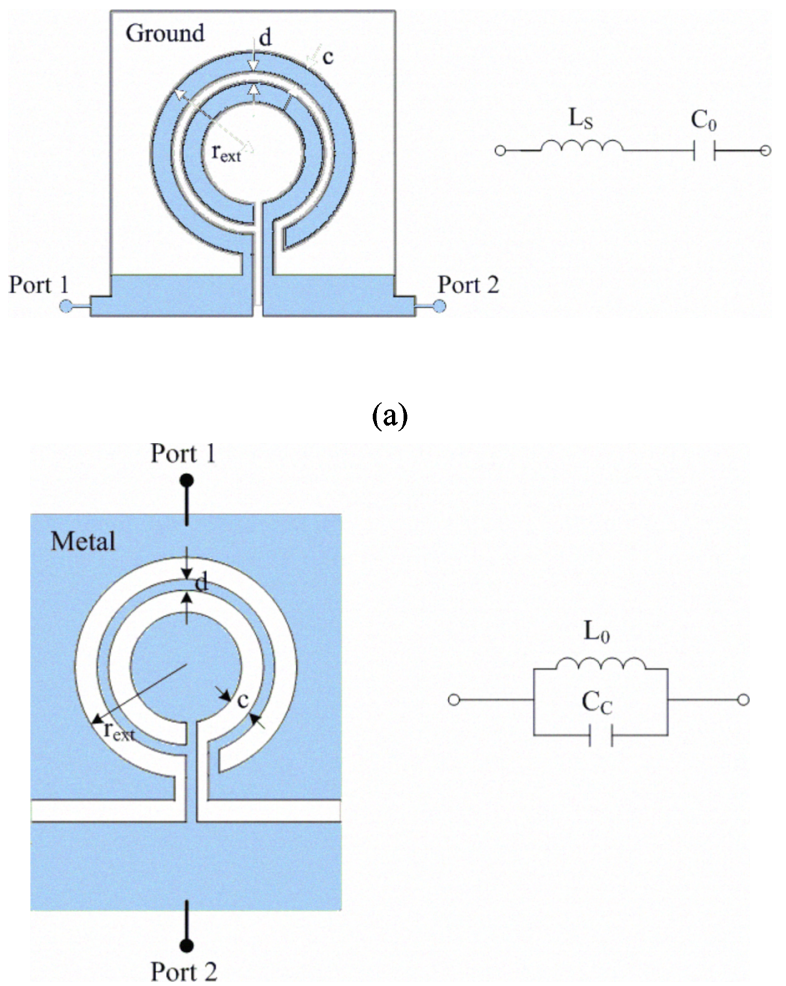

(b)

Fig. 2. Conventional OSRR and OCSRR structures with their lumped-element circuit models.

\section{Proposed OSRR and OCSRR resonators}

In our design, in order to enhance the coupling and increase the inductance of the structure, the split-ring gap, $\mathrm{d}$, is chosen to be the minimal available in the CMOS process. And the metallic ring strips have been designed as a meandering structure (Fig. 3). The width of the feeding transmission line is $18 \mu \mathrm{m}$, which is calculated by the transmission line theory for maximum power transfer.

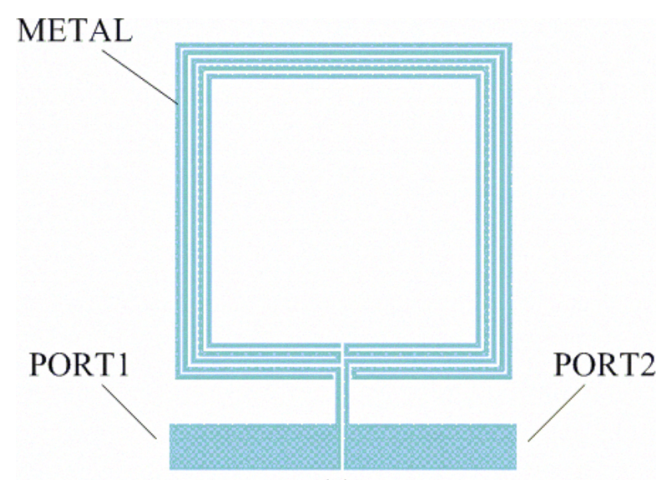

(a) 


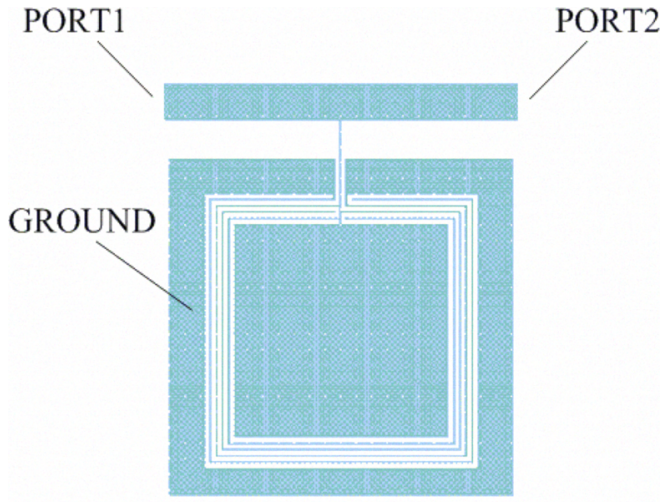

(b)

Fig. 3. Proposed on-chip OSRR and OCSRR structures.

The structures in Fig. 3 have three physical differences compared with the conventional ones in Fig. 2. Firstly, circular loops are replaced by square loops which are well compatible to the standard CMOS processes. Secondly, more than two square loops, which are formed by the two original paths turning back at the end, are employed compared with only two loops in Fig. 2. Although this introduces additional loss owing to the discontinuities at the turnings, the inductance can be increased, so that the resonance frequency will fall into the expected frequency band. Thirdly, in order to reduce the transfer loss and simultaneously simplify the structure, the employment of through-vias is avoided on the loops. In addition, because all the structures are planar, without any 3-D objects, it makes the system easy to analyze.

The frequency response of OSRR and OCSRR are shown in Fig. 4, which are bandpass and bandstop, respectively. From the models reported in [6], we can obtain the inductance and capacitance for the OSRRs and OCSRRs printed in the structure of Fig. 3. These values can be introduced into the lump-element models for circuit simulation which is basically consistent with the results simulated by EM simulators.

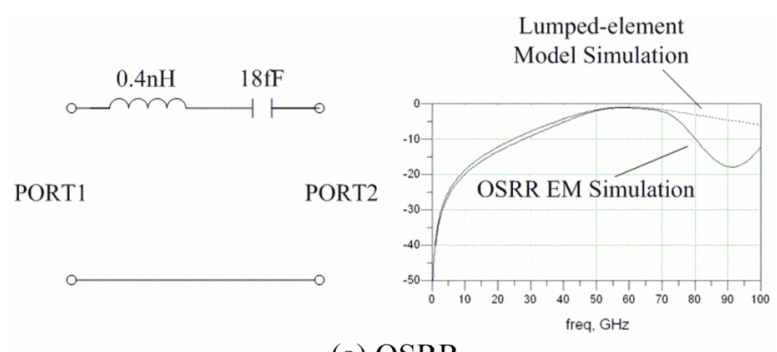

(a) OSRR

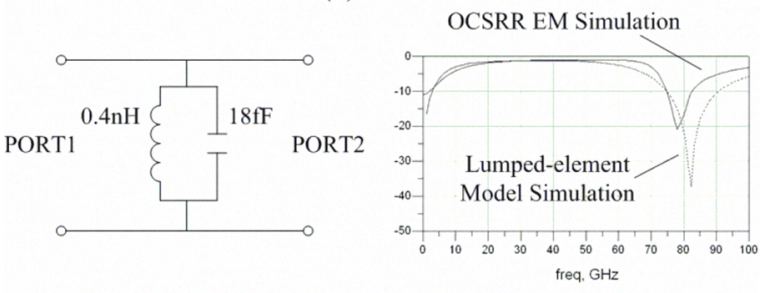

(b) OCSRR

Fig. 4. OSRR and OCSRR lumped-element models and their $\mathrm{S} 21$ responses.

\section{DESIGN EXAMPLE OF CMOS CRLH FILTERS}

An example 2-stage CMOS filter based on the CRLH structures is shown in Fig. 5. It is a $60-\mathrm{GHz}$ bandpass filter (BPF) consisting of two LC resonators in series, achieved by the proposed OSRR resonator.

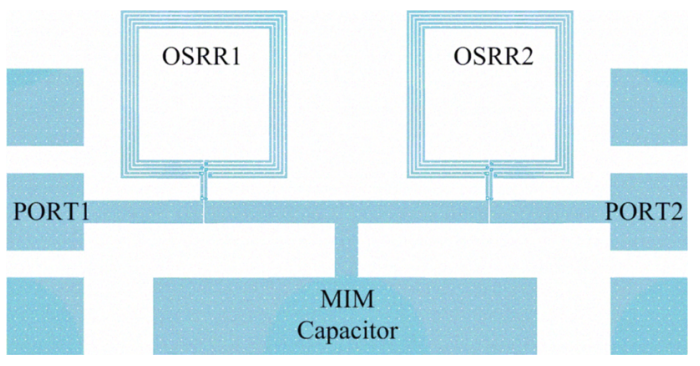

Fig. 5. Layout of the CRLH CMOS BPF.

In Fig. 5, the length of each side of the OSRR is approximately $100 \mu \mathrm{m}$. And the core dimension of the whole CRLH CMOS BPF is $350 \mu \mathrm{m}$ by $250 \mu \mathrm{m}$, which is smaller than any $60-\mathrm{GHz}$ passive BPF reported in the literature. It should be noted that, in the normal CMOS process, preventing the induced currents from being injected into the lossy silicon substrate is crucial. Therefore, the bottom metal (M1) is employed as the ground plane to shield and the top metal (UTM) is used as the resonator.

This structure is simulated by means of the electromagnetic simulator Agilent Momentum, on a Silterra $0.13 \mu \mathrm{m}$ CMOS substrate. The process layer parameters such as thickness and dielectric constants are all from the foundry datasheet. Conductor losses are modeled by using conductivity for copper. The simulated insertion and return losses for the structure are depicted in Fig. 6.

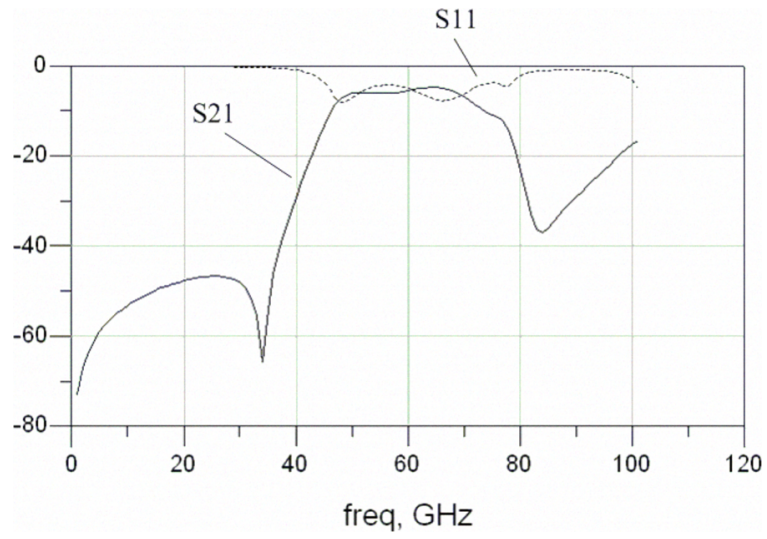

Fig. 6. S11 and S21 of the CRLH CMOS BPF.

It is obvious that the circuit has a bandpass characteristic around $60 \mathrm{GHz}$. The insertion loss is about $7 \mathrm{~dB}$ and the return loss is around $10 \mathrm{~dB}$. The $3 \mathrm{~dB}$ bandwidth of the implemented filter is about $15 \mathrm{GHz}$. The selectivity rejection is around $40 \mathrm{~dB}$ at $30 \mathrm{GHz}$. Compared 
with former CMOS BPFs, the proposed CRLH BPF has the advantages of smaller silicon size and better out-of-band rejection as shown in Table I.

TABLE I

Performance Comparison of CMOS BPFs

\begin{tabular}{|c|c|c|c|}
\hline Work & Freq. & Area & Rejection \\
\hline$[1]$ & $30 \mathrm{GHz}$ & $0.8 \times 0.6 \mathrm{~mm}^{2}$ & $-10 \mathrm{~dB} @ 15 \mathrm{GHz}$ \\
\hline$[2]$ & $60 \mathrm{GHz}$ & $0.79 \times 0.2 \mathrm{~mm}^{2}$ & $-15 \mathrm{~dB} @ 30 \mathrm{GHz}$ \\
\hline$[3]$ & $60 \mathrm{GHz}$ & $0.71 \times 0.71 \mathrm{~mm}^{2}$ & $-30 \mathrm{~dB} @ 50 \mathrm{GHz}$ \\
\hline Proposed & $60 \mathrm{GHz}$ & $0.35 \times 0.25 \mathrm{~mm}^{2}$ & $-40 \mathrm{~dB} @ 30 \mathrm{GHz}$ \\
\hline
\end{tabular}

\section{CONCLUSION}

A novel approach to design millimeter passive filters using CRLH structures on standard CMOS process is proposed. These filters employ OSRR or OCSRR as resonators and have the advantages of smaller silicon area and are easier to analyze based on simple lumped-element equivalent models.

\section{ACKNOWLEDGEMENT}

This work is supported in part by the Hong Kong Research Grants Council under Project HKU 717407E and in part by the University Research Committee of The University of Hong Kong.

\section{REFERENCES}

[1] C. H. Doan, S. Emami, A. M. Niknejad and R. W. Brodersen, "Design of CMOS for $60-\mathrm{GHz}$ applications," Proc. IEEE Solid-State Circuits Conf., 2004, pp. $440-$ 441.

[2] S. Sun, J. Shi, L. Zhu, S. Rustagi and K. Mouthaan, "Millimeter-wave bandpass filters by standard $0.18-\mu \mathrm{m}$ CMOS technology," IEEE Electron Device Lett., vol. 28, no. 3, pp. 220 - 222, Mar. 2007.

[3] C.-Y. Hsu, C.-Y. Chen, and H.-R. Chuang, "A 60-GHz millimeter-wave bandpass filter using 0.18- $\mu \mathrm{m}$ CMOS technology," IEEE Electron Device Lett., vol. 29, no. 3, pp. 246 - 248, Mar. 2008.

[4] J. Bonache, F. Martin, I. Gil, J. Garcia-Garcia, R. Marques, and M. Sorolla, "Microstrip bandpass filters with wide bandwidth and compact dimensions," Microwave and Optical Technology Letters, vol. 46, no.4, pp. 343 - 346, August 2005.

[5] A. Lai, T. Itoh, and C. Caloz, "Composite right/left-handed transmission line metamaterials," Microwave Magazine, IEEE, vol. 5, pp. 34 - 50, Sept. 2004.

[6] J. D. Baena, J. Bonache, F. Martin, R. Marques, F. Falcone, T. Lopetegi, M. A. G. Laso, J. Garcia, I Gil and M. Sorolla, "Equivalent circuit models for split ring resonators and complementary split rings resonators coupled to planar transmission lines", IEEE Transactions on Microwave Theory and Techniques, vol. 53, pp. 14511461, April 2005. 